\title{
PAVEMENT ANOMALIES DETECTION AND CLASSIFICATION USING ENTROPIC TEXTURE SEGMENTATION AND SUPPORT VECTOR MACHINES
}

\author{
Georgios M. Hadjidemetriou ${ }^{1}$ and Symeon E. Christodoulou ${ }^{2}$
}

\begin{abstract}
Presented herein is a vision-based method for the detection of anomalies on roadway pavements, utilizing low-cost video acquisition and image processing of road surface frames collected by a smartphone (or camera) located on a vehicle moving in a real-life urban network, along with entropy-based texture segmentation filters, and support vector machine (SVM) classification. The proposed system, which has been developed in MATLAB, pre-processes video streams for the identification of video frames of changes in image-entropy values, isolates these frames and performs texture segmentation to identify pixel areas of significant changes in entropy values, and then classifies and quantifies these areas using SVMs. The developed SVM is trained and tested by feature vectors generated from the histogram and two texture descriptors of non-overlapped square blocks, which constitute images that includes "patch" and "no-patch" areas. The outcome is composed of block-based and image-based classification, as well as measurement of the patch area.
\end{abstract}

Keywords: pavement condition evaluation, road anomaly detection, vision-based, entropy, texture segmentation.

\section{INTRODUCTION}

In recent years several national and transnational roadway management programs, such as the USA's "LTPP" and the EU's "Ten-T" programs, have been put to action in an effort to improve on the condition of transport networks and to mitigate the effects of time and of heavy usage on these networks. In fact, several regional and international studies estimate the annual potential impacts of changes in roadway maintenance expenditures (as these impacts relate to vehicle operating costs, safety, the environment, and the wider economy) at billions of dollars worldwide (Chatti and Zaabar 2012, National Economic Council 2014, Gleave et al. 2014). With the aforementioned in mind, it is imperative that automated roadway pavement condition assessment technologies are employed, so that sustainable and efficient roadway network management systems are developed. A key function of such technologies should be the automatic roadway defects detection and classification.

The paper presents a vision-based method for the automated detection of pavement anomalies by use of low-cost (smartphone) technology, image segmentation via entropy texture filters, and object classification via support vector machines (SVM). The images classified as containing pavement anomalies by the use of entropic measures, are processed by another algorithm which classifies pavement patches, using SVM classification. In spite of the fact that patching is a pavement maintenance activity, it is

1 Doctoral Candidate, Department of Civil and Environmental Engineering, University of Cyprus, Nicosia, Cyprus, ghadji01@ucy.ac.cy

2 Associate Professor, Department of Civil and Environmental Engineering, University of Cyprus, Nicosia, Cyprus, schristo@ucy.ac.cy 
also a type of pavement distress since it does not completely restore the pavement to its initial condition. Pavement condition manuals evaluate patching based on the percentage of the examined pavement area that contains patches (Kay 1992) and use this information for rating the pavement condition. Further to this brief introduction the paper includes a literature review on the state of knowledge in automated roadway anomaly detection. The main characteristics of a case-study implementation are then presented and finally the results and efficiency of the proposed method are discussed.

\section{BRIEF LITERATURE REVIEW}

The automated detection of roadway pavement anomalies by use of low-cost technologies has been the focus of several research efforts in the past decade, with these efforts generally classified in two categories: vibration-based and vision-based methods. A brief summary of some of the vision-based approaches and of their findings is listed below.

In the work by Nejad and Zakeri (2011) an automated imaging system was described for distress detection in asphalt pavements. The work focused on comparing the discriminating power of several multi-resolution texture analysis techniques using wavelet, ridgelet and curvelet-based texture descriptors, and concluded that curvelet-based signatures outperform all other multi-resolution techniques for pothole distress, (yielding accuracy rates of 97.9\%), while ridgelet-based signatures outperform all other multiresolution techniques for cracking distress (accuracy rates of 93.6\%-96.4\%). Radopoulou and Brilakis (2015) presented an application of the Semantic Texton Forests (STF) algorithm for automatically detecting patches, potholes and three types of cracks in video frames captured by a common parking camera, reporting over $70 \%$ accuracy in all of the tests performed, and over $75 \%$ precision for most of the defects. Subsequently, Radopoulou et al. (2016) utilized video data collected from a car parking camera to detect defects in frames and classified detected defects according to their type and severity. The researchers reported that the initial identification of frames including defects produced an accuracy of $96 \%$ and approximately $97 \%$ precision.

Tsai et al. (2010) assessed the performance of six segmentation algorithms, associated with cracks, concluding that the dynamic optimization based method had the best performance amongst them. The pavement distress type of raveling was quantified by Mathavan et al. 2014 using a combination of two- and three-dimensional images. A computer-vision approach was the subject of the work by Koch and Brilakis (2011), who proposed a method for automated pothole detection by which an image was first segmented into defect and non-defect regions using histogram shape-based thresholding, and then the texture inside a potential defect shape was extracted and compared with the texture of the surrounding non-defect pavement in order to determine if the region of interest represents an actual pothole. The aforementioned camera-based pothole-detection method was subsequently extended by Koch et al. (2013) for assessing the severity of potholes, by incrementally updating a representative texture template for intact pavement regions and using a vision tracker to reduce the computational effort. Related was also the work by Jog et al. (2012) who used vision-based data for both 2D recognition and for 3D reconstruction, based on visual and spatial characteristics of potholes, while measured properties were used to assess the severity of potholes. In the work by Ryu et al. (2015), a pothole detection method was presented using various features in two-dimensional images. The proposed method first uses a histogram and the closing operation of a morphology filter to extract dark regions for pothole detection, and then candidate regions of a pothole are extracted with the use of features such as size and compactness. Finally, a decision is 
made on whether candidate regions are potholes with a comparison of pothole and background features.

To the authors' knowledge, the most efficient and accurate vision-based patch detection method was proposed by Radopoulou et al. 2013. The algorithm considers some criteria for patch length, width and area. Though, their method performs high accuracy detection results if there is only one patch whose entire area is included and occupies $10 \%$ - $40 \%$ of the image. At a later stage, Radopoulou and Brilakis (2015) improved their algorithm, by detecting a patch based on its closed contour and texture, and passing it to a vision-tracking algorithm in order to trace it in subsequent video frames. Nonetheless, some false positives appear in the case of shadows, while the patch should still occupy a predetermined proportion of the frame $(2.5 \%-65 \%)$.

\section{METHODOLOGY}

\subsection{Anomaly Detection Using Entropic Measures in Images and Streaming Video}

The proposed approach makes use of image texture segmentation with entropy texture filters, and has been implemented on MATLAB's computer vision toolbox. Entropy is a statistical measure of randomness, and an entropy filter can characterize the texture of an image by providing information about the local variability of the intensity values of pixels in an image. For example, in areas with smooth texture, the range of values in the neighbourhood around a pixel will be a small value; in areas of rough texture, the range will be larger. Similarly, calculating the standard deviation of pixels in a neighbourhood can indicate the degree of variability of pixel values in that region.

The entropy (E) of a grayscale image (I) is defined as $E=-\operatorname{sum}\left[\mathrm{p} .{ }^{*} \log 2(\mathrm{p})\right]$, where $\mathrm{p}$ contains the histogram counts of the intensity image. By default, entropy uses two bins for logical arrays and 256 bins for uint8, uint16, or double arrays. The entropy filter $(\mathrm{J}=$ entropyfilt(I)) of a grayscale image returns the array $(\mathrm{J})$, where each output pixel contains the entropy value of the 9-by-9 neighbourhood around the corresponding pixel in the input image I (Fig. 1). Thus, the entropy filter creates a texture image. For pixels on the borders of I, entropyfilt uses symmetric padding, where the values of padding pixels are a mirror reflection of the border pixels in I.

The steps used in the proposed entropy texture segmentation approach are as listed below, with Fig. 2 serving as a reference for the resulting image at each analysis step:

1. Read a video of the roadway pavement to be analysed.

2. For each video frame, convert it to grayscale image (Fig. 2a) and calculate the overall image entropy.

3. If the computed image entropy deviates from the running average, then presume that the image contains a pavement anomaly (manifested in the image as texture anomaly) and isolate it for further analysis.

o $\quad$ Create a texture image (Fig. 2b).

o Threshold the image to segment the textures (a threshold value of 0.8 is used as default value, for it is roughly the intensity value of pixels along the boundary between the textures). A function is also used to smooth the edges and to close any open holes in objects.

o Partition the entropy image (Fig. 2b) into a grid (in this case from 1920x1080 pixels to 32x18 blocks), and count the proportion of black-to-white pixels in each grid cell. 
Threshold this ratio (say at 80\%) and output the resulting image (Fig. 2c). White regions indicate texture anomalies.

o Rescale the threshold texture image (Fig. 2c) back to the original image dimensions and display the segmentation results, marking the corresponding image areas as pavement anomalies (Fig. 2d).

\begin{tabular}{|c|c|c|c|c|c|c|c|c|c|c|c|c|c|c|c|c|}
\hline 255 & 241 & 199 & 199 & 238 & 255 & 255 & 255 & 255 & 247 & 199 & & & & & & \\
\hline 241 & 196 & 255 & 255 & 203 & 234 & 255 & 255 & 255 & 206 & 254 & & & & & & \\
\hline 199 & 255 & 255 & 255 & 255 & 194 & 255 & 255 & 255 & 209 & 249 & & & & & & \\
\hline 200 & 255 & 255 & 255 & 255 & 194 & 255 & 255 & 255 & 209 & 249 & & & & & & \\
\hline 234 & 209 & 255 & 255 & 214 & 226 & 255 & 255 & 255 & 206 & 254 & & & & & & \\
\hline 255 & 228 & 194 & 194 & 224 & 255 & 255 & 255 & 255 & 250 & 194 & & & & & & \\
\hline 255 & 255 & 255 & 255 & 255 & 255 & 255 & 255 & 255 & 255 & 255 & & & & & & \\
\hline 255 & 255 & 255 & 255 & 255 & 255 & 255 & 255 & 255 & 255 & 255 & & & & & & \\
\hline 255 & 255 & 255 & 196 & 226 & 255 & 255 & 255 & 255 & 255 & 255 & & & & & & \\
\hline 255 & 255 & 240 & 213 & 187 & 255 & 255 & 255 & 255 & 255 & 255 & & & & & & \\
\hline 255 & 255 & 178 & 255 & 255 & 183 & 255 & 255 & 255 & 255 & 179 & & & & & & \\
\hline & & & & & & 2.251 & 2.619 & 2.566 & 2.345 & 2.045 & 2.221 & 2.413 & 2.481 & 2.481 & 2.245 & 2.394 \\
\hline & & & & & & 2.622 & 2.823 & 2.738 & 2.460 & 2.202 & 2.483 & 2.572 & 2.688 & 2.708 & 2.474 & 2.664 \\
\hline & & & & & & 2.576 & $\begin{array}{l}2.738 \\
\end{array}$ & 2.636 & 2.351 & 2.118 & 2.393 & 2.430 & 2.542 & 2.558 & 2.321 & 2.518 \\
\hline & & & & & & 2.355 & 2.476 & 2.366 & 2.095 & 1.885 & 2.148 & 2.197 & 2.310 & 2.325 & 2.148 & 2.349 \\
\hline & & & & & & 2.269 & 2.342 & 2.256 & 2.025 & 1.799 & 1.979 & 2.089 & 2.167 & 2.183 & 2.028 & 2.167 \\
\hline & & & & & & 2.285 & $\begin{array}{l}2.335 \\
\end{array}$ & 2.228 & 2.044 & 1.792 & \begin{tabular}{|l|l|}
1.823 \\
\end{tabular} & 1.968 & $\begin{array}{l}2.013 \\
\end{array}$ & $\begin{array}{l}1.938 \\
\end{array}$ & 1.782 & 1.923 \\
\hline & & & & & & 2.093 & 2.162 & 1.988 & 1.857 & 1.659 & 1.691 & 1.928 & 1.882 & 1.807 & 1.741 & 1.882 \\
\hline & & & & & & 2.246 & 2.335 & 2.149 & 1.954 & 1.822 & \begin{tabular}{ll|}
1.888 \\
\end{tabular} & 2.053 & 1.913 & 1.807 & 1.775 & 1.947 \\
\hline & & & & & & 2.349 & 2.404 & 2.302 & 2.053 & 1.922 & $\begin{array}{ll}1.922 \\
\end{array}$ & 1.913 & 1.832 & 1.716 & 1.775 & 1.997 \\
\hline & & & & & & 2.520 & 2.513 & 2.413 & 2.223 & 2.113 & 2.073 & 1.974 & 1.804 & 1.713 & 1.882 & 2.078 \\
\hline & & & & & & 2.030 & 2.094 & 2.055 & 1.925 & 1.813 & \begin{tabular}{ll|}
1.681 \\
\end{tabular} & 1.614 & 1.451 & 1.374 & 1.500 & 1.617 \\
\hline
\end{tabular}

Figure 1: Sample entropyfilt() calculations
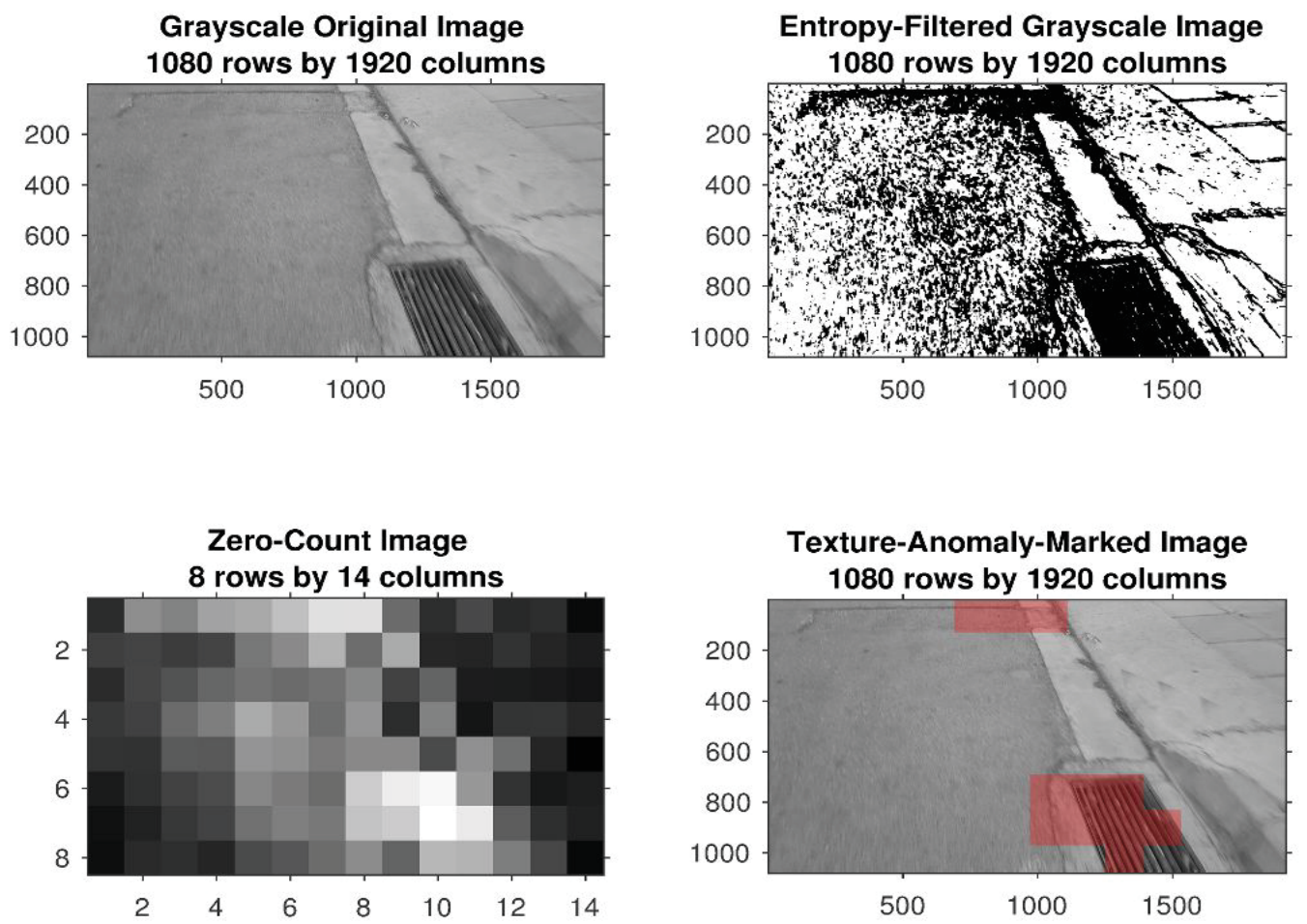

Figure 2: Sample texture segmentation and anomaly detection using entropy filters. 


\subsection{Patch Classification Using Support Vector Machines (SVMs)}

The texture segmentation approach has been complimented with support vector machines (SVMs) and applied to patch classification. SVMs are supervised machine learning models, which identify patterns after taking labelled training data. An SVM is divided into two main phases (training and testing), and it can be used efficiently for two-group classification problems such as the presented research study ("patch" vs. "no-patch" classes).

The images classified as containing a pavement anomaly by the previous procedure, are used by another algorithm, which divides them between those that include the pavement distress type of patch and those that do not; saving a significant amount of time since the new algorithm has a smaller number of frames to process. A multiclass classification algorithm can be developed in future work to categorise even more forms of pavement distress, utilizing the frames characterised as including a pavement anomaly by the aforementioned algorithm. The key steps of the presented algorithm (Hadjidemetriou et al. 2016) are presented in Fig. 3 for both the training and testing stages. The SVM training phase begins with transforming collected pavement video frames into grayscale images. The presented system uses only one SVM, which is trained by labelled data (ground truth) and feature vectors.

The ground truth is entered in the algorithm given data regarding the pixels of each frame which are part of a pavement patch. Each feature vector is generated, and subsequently the SVM is trained, extracting information from non-overlapped areas within the frame, whose size is 20x20 pixels in width and height. The selection of blocks size is based on usual image resolutions, whose dimensions are multiples of 20 (e.g. 640x480), so that blocks would cover the whole image. A number of block sizes, which fulfil this criterion (e.g. 10x10), have been tested and the use of a trial and error technique designates our final district size (20x20). One should also note that blocks which are comprised of weighty proportions of both patch and non-patch areas (i.e. the patch area is more than $5 \%$ and less than $95 \%$ of the block) are not used to facilitate the training of the SVM and consequently its ability to distinguish "patch" from "no-patch" areas. Every feature vector, corresponding to a square block, is generated by the local intensity histogram and two texture descriptors, named two-dimensional Discrete Cosine Transform (DCT) and Gray-Level Co-occurrence Matrix (GLCM). DCT, which can be used efficiently for purposes of pattern recognition, expresses a finite amount of data points in respect of a weighted sum of cosine functions oscillating at diverse frequencies. GLCM is a statistical system that examines the spatial relationship of pixels; while its functions are able to designate the texture of a picture by creating a matrix, which contains the estimated frequencies of the occurrence of pixel pairs with definite values and in a specific spatial relationship. The presented method extracts data from this matrix to calculate and then use the statistical measures of contrast, correlation, energy and homogeneity.

The SVM training stage is followed by a testing phase (Fig. 3b). Its flow is similar with the SVM training, starting with transforming RGB pavement frames into grayscale images and dividing them into square blocks of $20 \times 20$ pixels. A feature vector is formed by the local intensity histogram and the two texture descriptors for each square block. The flowchart continues with the feature vector used by the SVM to classify each block of the testing pavement picture in "patch" (1) or "no-patch" (0) categories. Fig. 4 depicts the way patch areas are identified by the algorithm; where yellow-colored blocks represent the "patch" class and blue-colored cells correspond to the "no patch" group. 

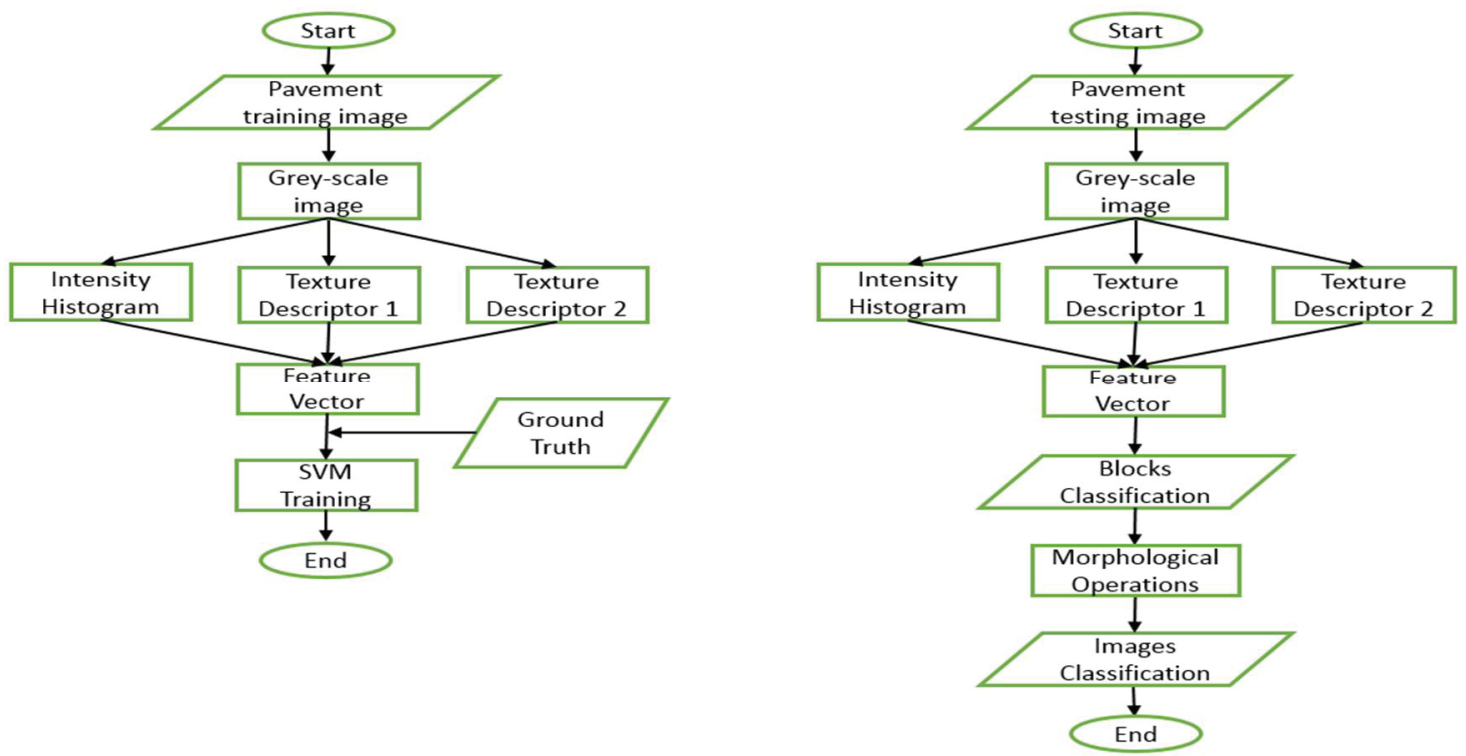

Figure 3: Training and testing stages of the proposed patch detection algorithm.
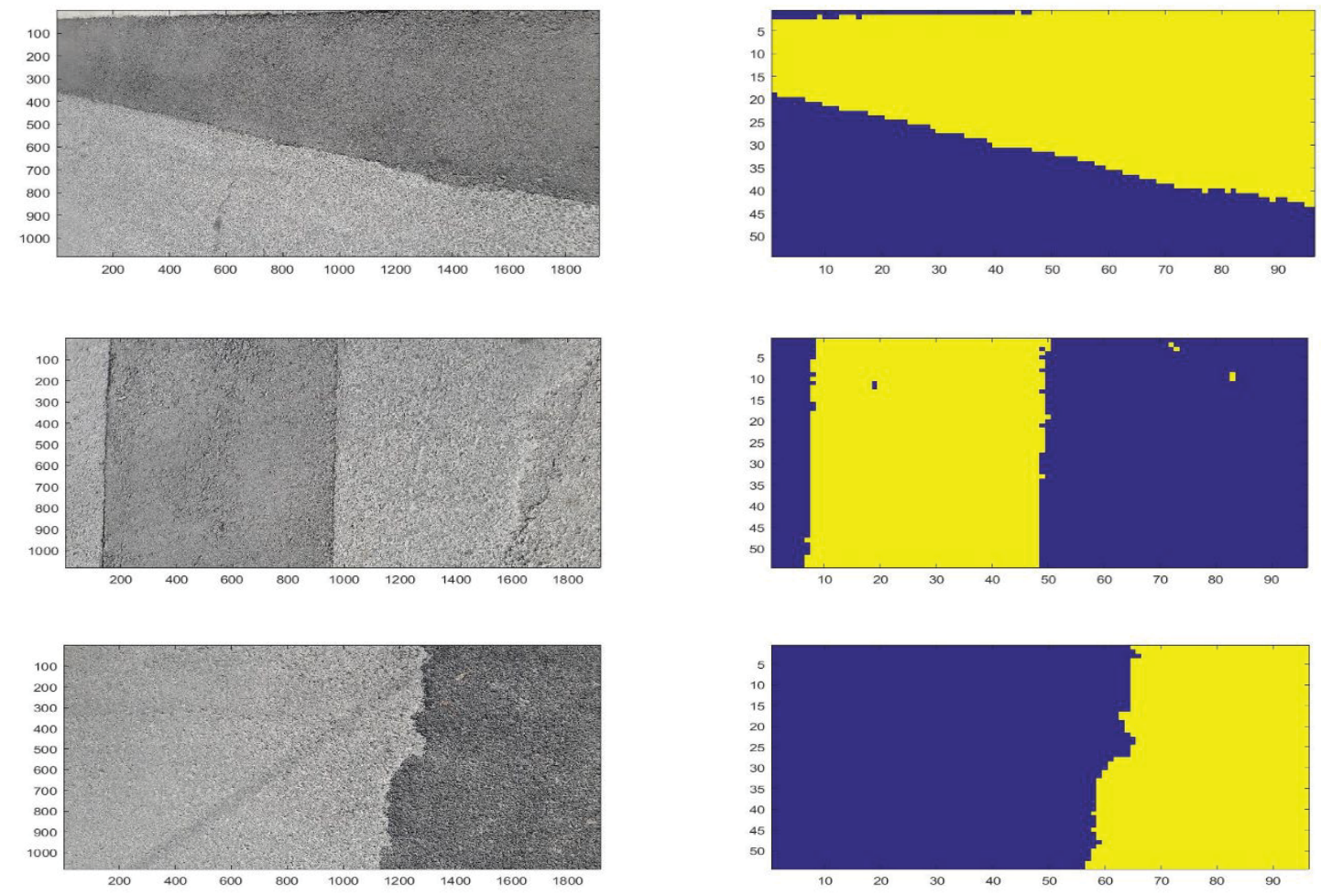

Figure 4: Examples of processed images by the proposed algorithm.

Further, the morphological operation of closing is applied, to fill and eliminate blocks which are classified differently than their surrounded blocks, by changing their label from 0 to 1 and vice versa. Finally, a trial and error technique is used to define the number of connected "patch" blocks (340) which indicate the presence of a patch in an image. Consequently, the algorithm, after the blocks classification, discriminates between the images which include parts of patches and the frames which do not contain any patches 
parts (image classification). At this point, the difference between presence and detection should be clarified. The former answers with a "yes" or a "no" the question of whether an examined object occurs in an image, while the latter provides information regarding the place of the object in the image. Despite the restriction of a range of algorithms to only identifying the presence of a distress, while classifying images between damaged and undamaged pavement, the proposed algorithm achieves both the presence and detection of patches.

The method has been successfully field-tested on case-study roadways, using either a GoPro camera or a smartphone camera. The vehicle moved with an average speed of 20 $\mathrm{km} / \mathrm{h}$. The external camera was a GoPro HERO3+ with a wide-angle lens. It was positioned at a height of $1.00 \mathrm{~m}$ above the ground. In addition, a 13 MP Smartphone camera (Samsung A5-2016) collected video frames of the roadway from within the vehicle. Table 1 presents the evaluation of the method performance in terms of accuracy, precision and recall. The appearance of False Positives, which affect accuracy and precision, is mainly caused by shadows and in some cases by oil spots. The performance is lower when data is collected by the smartphone. This might be interpreted by the fact that frames are affected by the cleanness of the window, captured objects outside of the area of interest and the greater distance between camera and pavement, compared to the other scenario.

Table 1: The performance of blocks and images classification - (a) GoPro camera;

(b) smartphone camera.

\begin{tabular}{cccccccc}
\hline Blocks Classification & Images Classification & \multicolumn{3}{c}{ Blocks Classification } & \multicolumn{2}{c}{ Images Classification } \\
\hline & (GoPro camera) & \multicolumn{5}{c}{ (smartphone camera) } \\
\hline Accuracy & $82.9 \%$ & Accuracy & $82.5 \%$ & Accuracy & $80.5 \%$ & Accuracy & $80.0 \%$ \\
Precision & $65.6 \%$ & Precision & $77.8 \%$ & Precision & $63.8 \%$ & Precision & $75.4 \%$ \\
Recall & $92.0 \%$ & Recall & $91.0 \%$ & Recall & $89.4 \%$ & Recall & $89.0 \%$ \\
\hline
\end{tabular}

\section{CONCLUSIONS}

The paper presented a texture segmentation method with SVM classification for the detection of roadway anomalies (herein focused on patch detection), exhibiting high detection accuracy levels (above $80 \%$ ). The performance results are slightly better when a camera is positioned on the rear of car, compared to the placement of a smartphone camera inside a vehicle. The proposed method is also characterized by some strong advantages such as the identification of multiple patches in a single image or the detection of proportions of patches when their entire area is not included in the image. Ongoing work includes the accurate measurement of the pavement patch area, and the inclusion of other roadway defects in the classification process.

\section{REFERENCES}

Chatti, K. and Zaabar, I. (2012). Estimating the effects of pavement condition on vehicle operating costs. Vol. 720. Transportation Research Board. 
Gleave, S. D., Frisoni, R., Dionori, F., Casullo, L., Vollath, C., Devenish, L., Spano, F., Sawicki, T., Carl, S., Lidia, R., Neri, J., Silaghi, R. and Stanghellini, A. (2014). EU road surfaces: Economic and safety impact of the lack of regular road maintenance. European Parliament - Directorate General for Internal Policies, Policy Department B: Structural and Cohesion Policies, Transport and Tourism.

Hadjidemetriou, G.M., Christodoulou, S.E. and Vela, P.A (2016). Automated detection of pavement patches utilizing support vector machine classification. In Proc. Electrotechnical Conference (MELECON), 2016 18th Mediterranean (pp. 1-5), IEEE.

Jog, G., Koch, C., Golparvar-Fard, M. and Brilakis, I. (2012). Pothole properties measurement through visual $2 \mathrm{~d}$ recognition and $3 \mathrm{~d}$ reconstruction. In Proc., ASCE International Conference on Computing in Civil Engineering, 553-560.

Kay, R. K. (1992). Pavement surface condition rating manual. Washington State Transportation Center, University of Washington.

Koch, C. and Brilakis, I. (2011). Pothole detection in asphalt pavement images. Advanced Engineering Informatics, 25(3):507-515.

Koch, C., Jog, G. and Brilakis, I. (2013). Automated pothole distress assessment using asphalt pavement video data. Journal of Computing in Civil Engineering 27(4):370378.

Mathavan, S., Rahman, M., Stonecliffe-Jones, M. and Kamal, K. (2014). Pavement raveling detection and measurement from synchronized intensity and range images. Transportation Research Record: Journal of the Transportation Research Board, 2457, 3-11.

National Economic Council, The President's Council of Economic Advisers (2014). An economic analysis of transportation infrastructure investment. The White House, Washington DC.

Nejad, F. and Zakeri, H. ( 2011). A comparison of multi-resolution methods for detection and isolation of pavement distress. Expert Systems with Applications 38(3):2857-2872.

Radopoulou, S. and Brilakis, I. (2015). Detection of multiple road defects for pavement condition assessment. In Proc., EG-ICE 2015 - 22nd Workshop of the European Group of Intelligent Computing in Engineering.

Radopoulou, S. C. and Brilakis, I. (2015). Patch detection for pavement assessment. Automation in Construction, 53, 95-104.

Radopoulou, S. C., Jog, G. M., Brilakis, I., Radopoulou, S. C., Jog, G. M. and Brilakis, I. (2013). "Patch distress detection in asphalt pavement images." ISARC Proceedings, 2013 Proceedings of the 30th ISARC, Montréal, Canada, 1572-1580.

Radopoulou, S., Brilakis, I., Doycheva, K. and Koch, C. (2016). A framework for automated pavement condition monitoring. In Proc., Construction Research Congress 2016: Old and New Construction Technologies Converge in Historic San Juan - Proceedings of the 2016 Construction Research Congress, CRC 2016, 770- 779.

Ryu, S.-K., Kim, T. and Kim, Y.-R. (2015). Feature-based pothole detection in twodimensional images. Transportation Research Record 2528:9-17.

Tsai, Y.-C., Kaul, V. and Mersereau, R. M. (2010). Critical assessment of pavement distress segmentation methods. Journal of Transportation Engineering, 136(1), 11-19. 